\title{
LOS PROCESOS DE DESARROLLO LOCAL Y EL FOMENTO DE LA EMPRESARIALIDAD: EXPERIENCIAS A PARTIR DEL ANÁLISIS \\ EN LA INDUSTRIA MUEBLERA DEL ESTADO DE JALISCO
}

\author{
Katia Magdalena Lozano Uvario \\ CUCSH \\ UNIVERSIDAD DE GUADALAJARA
}

\begin{abstract}
RESUMEN
El presente documento se enfoca al análisis de la participación del ámbito empresarial y la empresarialidad en los procesos de desarrollo local. Para ello se consideran tres apartados: el primero plantea las razones por las que se considera el ámbito económico como uno de los ejes del desarrollo local, resaltando el papel de las micro y pequeñas empresas así como de los sistemas productivos locales en la integración de las relaciones socioterritoriales que promueven el desarrollo de un territorio; en el segundo apartado se define la empresarialidad y se explica por qué su fomento constituye una tarea correlativa a la práctica del desarrollo local; por último, se analiza el caso de la industria mueblera en Jalisco como ejemplo de la promoción de la empresarialidad enfocada a incrementar la competitividad y la supervivencia de un sector tradicional.
\end{abstract}

Palabras clave: empresarialidad, desarrollo local, industria mueblera. 


\begin{abstract}
This article is focused on analyzing the roles played by the business sphere and entrepreneurship in local development processes. The first of the article's three sections proposes the reasons for which the economic sphere is considered to be one of the main focuses of local development, with emphasis on the role of micro and small-sized businesses as well as local productive systems in socio-territorial relationships that promote a territory's development. In the second section, the author defines entrepreneurship, and explains why promoting entrepreneurship is a task that is correlated with local development practice. The third section analyzes the case of Jalisco's furniture industry, as an example in which entrepreneurship is promoted, with emphasis on increasing competitiveness and the survival of a traditional economic sector.
\end{abstract}

Key words: entrepreneurship, local development, furniture industry. 


\section{EL ÁMBITO EMPRESARIAL EN EL DESARROLLO LOCAL}

De las distinciones en el estudio de los procesos de desarrollo local, la consideración del ámbito empresarial constituye uno de los ejes o puntos centrales para su tratamiento, así como para las formas en las cuales podemos reconocer este tipo de dinámicas. De hecho, los aspectos económicos tienen un sitio privilegiado en las estrategias de desarrollo local, ya que han aportado elementos de identidad a esta perspectiva teórico-práctica al centrarse en los recursos endógenos y en el apoyo preferente a las micro y pequeñas empresas (Carrillo 2002: 16).

Desde los primeros referentes teóricos del desarrollo local, como los propuestos por Arocena (1995) y Vázquez (1999), se distingue la incorporación del sistema económico, la dimensión económica, al apuntar que cuando de lo que se trata es de interpretar los procesos de desarrollo y, por tanto, las relaciones entre cambio estructural y espacial e innovación, los agentes económicos más deseables son las empresas, las organizaciones empresariales que siguen preferentemente los modelos de organización flexible de la producción, como son las redes de empresas de alta tecnología o los sistemas locales de empresa (Vázquez 1999: 91-92).

En lo particular, al retomar las ideas de Coffey, Polese y Stöhr, identifica Vázquez (1988: 26) como actores para la dimensión económica a los empresarios locales, quienes emplean su capacidad para organizar los factores productivos locales con niveles de productividad suficiente para ser competitivos en los mercados. Asimismo señala que las políticas de desarrollo local, a partir de las características de la estructura productiva, deben proponerse, con el fin de mejorar la capacidad empresarial y organizativa, la calidad de los factores y la difusión de las innovaciones en el tejido productivo y en los territorios, de 
igual manera la calidad del capital humano y la flexibilidad del sistema productivo (Vázquez 2001: 47-55).

Por otra parte, una característica trascendente de este tipo de desarrollo es que incluye como punto nodal la visión sistémica (Alburquerque 2005) ${ }^{1}$, esto se refiere a que el sistema empresarial se vincula con el entorno y por consiguiente se inserta en los tejidos sociales, comprometiéndose por ende con el destino de su espacio de acción. En este sentido, el desarrollo local modifica la perspectiva tradicional del desarrollo económico, y a la vez señala que el estudio de los procesos y las estrategias de desarrollo debe incluir además de las acciones de fomento al crecimiento económico la potenciación de los recursos endógenos del territorio, dando con ello valor a las dimensiones sociales, ambientales y culturales que permiten mejorar los niveles de bienestar de la sociedad. Es decir, el desarrollo local es concebido como el resultado de la influencia conjunta tanto de factores económicos como extraeconómicos que además presentan un carácter localizado, inherente a cada territorio.

Ahora bien, un papel relevante en el sistema de acción empresarial es el que se confiere a la pequeña empresa, principalmente por su poder de trasformación en pro del desarrollo local (Arocena 1995). Se afirma que la empresa de menor tamaño tiene a su favor, entre otros: la flexibilidad de su estructura y su capacidad de adaptación; su facilidad en la generación de empleo así como de un tejido empresarial que puede trascender a formar redes innovadoras, en las cuales la incorporación de la subcontratación les da la posibilidad de conformar en el mediano y largo plazo procesos de desarrollo sostenido; también el impulso a las micro y pequeñas empresas trae consigo el nacimiento de iniciativas de economía social y de autoempleo (Carrillo 2002). 
No obstante lo anterior, de igual manera es cierto que el pequeño empresario en muchas ocasiones vive como un «actor aislado», por las dificultades que tiene de insertarse en las redes comerciales y en los círculos financieros (Arocena 1995), por lo que la organización gremial y el apoyo institucional juegan un papel relevante en propiciar la articulación productiva y la existencia de un entorno empresarial que favorezca el desarrollo de los negocios (Cortelesse 1993).

De esta manera el éxito de una empresa no se relaciona sólo con el dinamismo del sector al que pertenece o con una capacidad interna superior para inventar nuevas soluciones, sino que en el intento de adaptarse para dar respuestas a las exigencias del mercado la empresa también se sirve del patrimonio de experiencias y de relaciones del sistema local al que pertenece (Sforzi 2001: 28). Por ello, los sistemas productivos locales se analizan a partir del entorno local que integran y en el cual destacan las reglas, normas y valores que rigen al sistema, así como el entramado de relaciones que se basan precisamente en el conocimiento que los actores tienen de los otros, en la confianza mutua que se ha generado de manera paulatina, y en el beneficio que reportan los intercambios económicos (Ottati 1994, citado en Vázquez 1999).

En suma, el análisis de los sistemas productivos locales, con el fin de cubrir los ámbitos que abarca el desarrollo local, debe incluir lo siguiente:

1) El sistema empresarial, del cual parte el análisis e incluye de manera directa a los actores empresariales de las cadenas productivas — productores, proveedores y comercializadores-; y los mecanismos de reconversión industrial, planteados para dinamizar su territorio, 
2) El sistema político-administrativo, que implica la revisión de la política económica local; y el aparato institucional, que hace referencia a los organismos vinculados con el sector productivo, tales como las cámaras o asociaciones empresariales, los organismos gubernamentales de fomento en los diferentes niveles de gobierno...

3) El sistema socioterritorial, en el cual se debe analizar el impacto de la identidad de los actores locales en sus territorios; es decir, la cultura productiva, los patrones de localización y las estrategias territoriales, incorporando la historia productiva del sistema (Lozano y Méndez 2002).

\section{LA EMPRESARIALIDAD Y LA PRÁCTICA DEL DESARROLLO LOCAL}

La empresarialidad es entendida como la capacidad para generar y crear nuevas iniciativas empresariales. Es señalada como uno de los aspectos que contribuyen al desarrollo económico, entre otras razones, por la generación de nuevos puestos de trabajo, aumento de la productividad, rejuvenecimiento de los tejidos socioproductivos, y por la producción de nuevas ideas e innovación (Kantis 2002, Viego 2004).

Desde la visión del desarrollo local, la empresarialidad se integra dentro de los elementos que reciben atención prioritaria, pues es vista como un factor dinámico que se orienta a la creación de empleo en un contexto en el que las nuevas formas de organización de la producción impulsan fuertes desplazamientos laborales (OCDE 1998a, en Viego 2004); y como alternativa intenta reconciliar el crecimiento económico con la cohesión social (Viego 2004). Por ello, debido a que el desarrollo local es considerado como una posición teórico-práctica que intenta dar respuestas a las crisis de desempleo que se 
generaron en los países desarrollados a finales de los años setenta del siglo XX, y luego como una «respuesta de solidaridad» que surgía en colectividades, regiones fragilizadas y marginadas que rechazaban la exclusión (Vachon 2001); la empresarialidad se apuntala como una de las estrategias de la política de desarrollo local que, siguiendo las ideas propuestas por Vázquez (1993: 228; 2001: 47-55), tratan de suplir las carencias y mejorar los factores inmateriales del desarrollo, así como fortalecer la capacidad organizativa del territorio.

Por su parte, en lo que corresponde al desarrollo empresarial que fomenta la regeneración del tejido productivo, la innovación es el componente básico de las estrategias que mejoran las capacidades de emprender y de gestionar a través del sistema productivo, a partir no solo de la introducción de innovaciones en procesos, productos y organización, sino también mediante la mejora del conocimiento — capacitación de recursos humanos-, que a la vez desarrolle una adecuada capacidad empresarial y organizativa. Ambos factores son estratégicos en la trasformación y modernización de los sistemas productivos locales y por ende del desarrollo económico de las regiones (Vázquez 2005).

Entre los factores reconocidos que inciden en la empresarialidad se encuentran por un lado aquellos señalados como triviales, definidos así por reunir las condiciones básicas para ingresar y prosperar en la actividad económica; estos son la motivación, el acceso a los recursos y capacidades —densidad y tasa de urbanización, tasa de escolaridad, disponibilidad de información, estructura productiva, ahorro local y funcionamiento del mercado de capitales-, y los factores sectoriales junto con las condiciones macro económicas; por otro lado, se añaden los factores que dependen del entorno institucional (Viego 2004). 
Al respecto, Kantis también plantea un «sistema de desarrollo emprendedor» (2004a: 28), el cual incorpora un conjunto de factores que contribuyen a comprender la naturaleza compleja y contextual del proceso emprendedor, afectando los siguientes aspectos: la formación de la motivación y las competencias para emprender la cultura y el sistema educativo, con elementos tales como las condiciones socioeconómicas de los hogares de los potenciales emprendedores; y la identificación de oportunidades de negocios, la elaboración del proyecto y la decisión de emprender, donde las condiciones macro económicas, la estructura dinámica y productiva, el acceso a los recursos, inciden en las expectativas y las oportunidades para crear o no empresas. Mención especial requieren las regulaciones y políticas que afectan la gestión del desarrollo de la empresa a partir de definir, por ejemplo, los trámites necesarios para formalizar una nueva o los impuestos, entre otros (ver Figura 1).

Figura 1. Sistema de desarrollo emprendedor

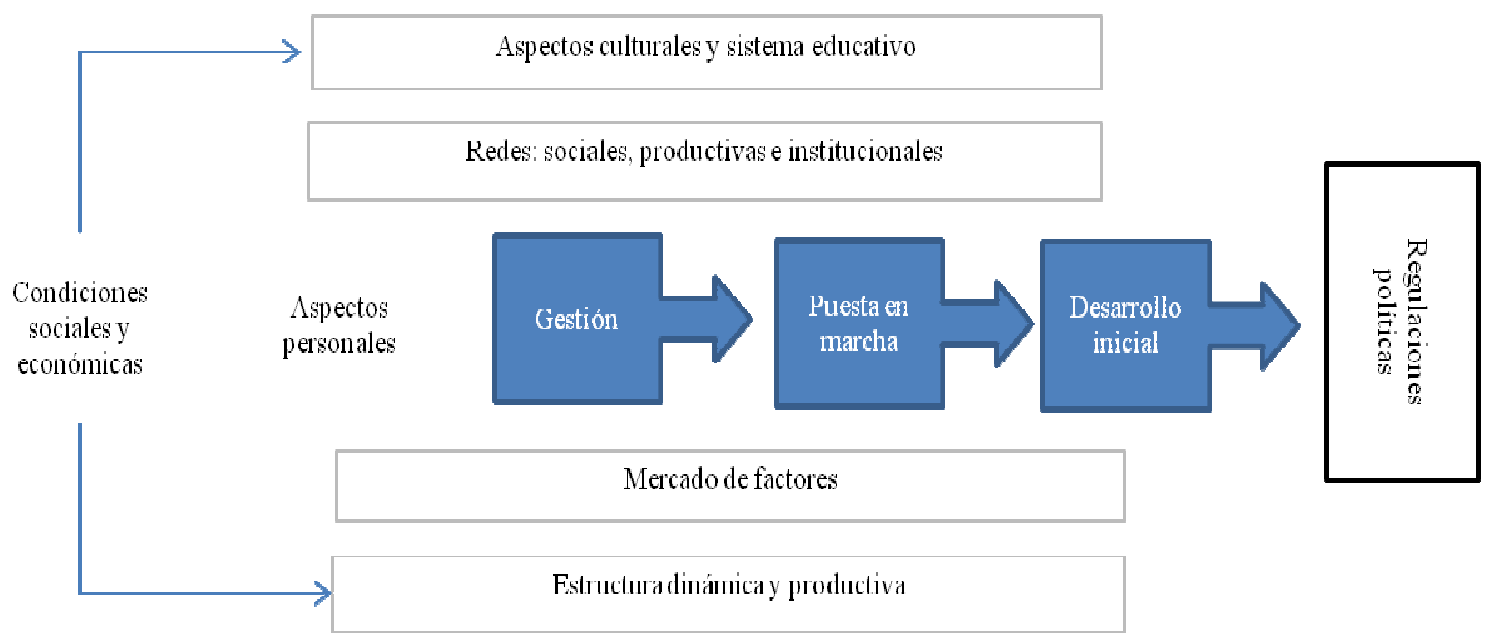

Fuente: Kantis 2004a: 28. 
De lo anterior se desprende la gran importancia que tiene la formación de un entorno para el surgimiento y desarrollo de la empresarialidad, donde las instituciones juegan un papel central en los procesos de crecimiento económico y de ajuste estructural al afectar las oportunidades de negocios y el acceso a ellas, la adquisición de vocaciones y competencias, así como el ingreso al mercado (Kantis 2004a).

Al respecto, Giovanni Stumpo comenta la acción que las instituciones ${ }^{2}$ pueden desempeñar en el fortalecimiento de los procesos de articulación entre las empresas, pues no solo contribuyen a la resolución de problemas específicos sino también se convierten en el lugar a través del cual se canalizan las acciones de cooperación horizontal entre empresas, y por tanto contribuyen a generar mayor confianza entre ellas (Stumpo 2004: 19). En este mismo sentido la teoría de la proximidad ha establecido que las instituciones son el vehículo que facilitan la interacción de las organizaciones entre sí y con el territorio, por lo que serían el eje a través del que se articulan los procesos territoriales de crecimiento y acumulación de capital (Vázquez 2005: 128).

En América Latina, la formación de instituciones se ha dado, por ejemplo, en la época del cambio en el modelo de producción, de la sustitución de importaciones a la apertura de mercados; lo que llevó consigo la trasformación de las comunidades latinoamericanas a un entorno de mayor apertura y competitividad, de esta manera las obligó a crear instancias institucionales, tanto de carácter gubernamental como de organización empresarial, que permitieran diseñar las nuevas estrategias del desarrollo (Ruiz 2005).

En este caso es importante resaltar aquellas que han destacado la empresarialidad, pues además de mantener la actividad económica fomentan la construcción de nuevas 
capacidades productivas competitivas. Adicionalmente, la elaboración de diálogos para impulsar estrategias de más largo alcance para las comunidades ha permitido la organización empresarial a través de cámaras empresariales, que se pueden convertir en verdaderas promotoras del desarrollo al vincular la vida empresarial con la sociedad civil.

Méndez (2000) sostiene que las asociaciones empresariales, en especial las de fuerte implantación local y ámbito de actuación sectorial, por su propio origen y el conocimiento directo de los asociados, contribuyen a reducir la desconfianza mutua —aunque su representatividad en ocasiones sea débil por la escasez de socios, de recursos materiales y humanos- En este sentido, resalta el elemento confianza como un factor importante que permite en menor o mayor medida afianzar el entramado de relaciones que se tejen entre los actores del sistema productivo y en sí la cooperación.

Aun con lo comentado, no todas las comunidades y los sistemas productivos locales basan sus relaciones de cooperación en la confianza; a la vez, lo relevante resulta el apoyo que deben ejercer las instituciones, enraizadas en la cultura local, para que confluyan las acciones y estrategias de las empresas y los actores en el territorio. Aunque también es importante señalar que muchas organizaciones empresariales no han funcionado como se hubiera deseado, en virtud de la falta de información por parte del empresario de la existencia de éstas, además de un nulo interés generado por la desconfianza que se tiene del gobierno, y el grave problema de ver a los empresarios del mismo gremio como competidores y no como socios (Ruiz 2002a: 137).

Otros actores institucionales importantes que se reconocen en la escena local son los centros tecnológicos y los gobiernos locales. Los primeros centran su atención en la realización de actividades de investigación y desarrollo, I+D, en la prestación de servicios 
técnicos demandados por las empresas del sector, y en la difusión de información útil para los empresarios y la promoción de cursos de formación, a menudo en colaboración con otros agentes. Por su parte, los gobiernos locales incorporan la promoción del desarrollo dentro de los objetivos prioritarios de su gestión, mediante el fomento del empleo, la mejora de infraestructuras y servicios tecnológicos, la creación de un clima de concertación y el fomento de la industria local (Méndez 2000).

En la práctica, es decir, en la generación de estrategias y políticas de desarrollo local, la empresarialidad involucra tanto a los actores empresariales como a los institucionales, a partir de las acciones de promoción y fomento. Específicamente, las políticas dirigidas a promover la empresarialidad se enfocan en los apoyos a la capacitación y a la educación como elementos que motivan el surgimiento de nuevas empresas, en virtud de que la experiencia laboral, más que la educación, la familia o los empresarios ejemplares, es el factor que tiene un impacto mayor en la generación de motivación y habilidades para emprender (Angelelli y Llisterri 2003).

Asimismo se apunta que las redes pueden facilitar el acceso a oportunidades de negocio, información, tecnología, y también la solución de los problemas del desarrollo inicial de las empresas (Ibíd.). La interacción social y comercial con otras personas $-\mathrm{y}$ empresas- es de los principales recursos del emprendedor. En este sentido, la articulación en el corto y largo plazo debe incluir una estrategia integral que construya alianzas capaces de agregar valor y complementar las capacidades y las experiencias en torno a una visión estratégica compartida (Kantis 2004b).

En este mismo orden, las iniciativas de la empresarialidad se pueden clasificar definiendo el tipo de intención que presentan: algunas buscan ensanchar o desplazar las 
fronteras del espacio emprendedor, facilitan el acceso a los mercados —a través, por ejemplo, de la promoción de exportaciones— y la promoción de los negocios innovadores; mientras que otras alternativamente promueven una mayor explotación de las oportunidades y los recursos existentes dentro de las fronteras actuales, a partir, por ejemplo, de la asistencia técnica y financiera a proyectos emprendedores (Kantis 2004b).

De lo expuesto con anterioridad se advierte que una clave en la práctica del desarrollo local y la empresarialidad es diagnosticar cuáles son los mecanismos óptimos para el desarrollo empresarial, mismos que dependerán tanto del lugar y sus condiciones estructurales —económicas, sociales, políticas y culturales- como del sistema productivo de que se trate, además de la manera en que se encuentre estructurado y los vínculos que tenga o necesite con su entorno. Como ejemplo de lo anterior, en el siguiente apartado se presentan las acciones iniciadas para el fomento del sistema productivo y la empresarialidad en la industria mueblera en Jalisco.

\section{LA INDUSTRIA MUEBLERA EN EL ESTADO DE JALISCO: EL FOMENTO DEL SISTEMA PRODUCTIVO Y LA EMPRESARIALIDAD}

La industria mueblera en Jalisco se ha caracterizado por tener una presencia constante dentro de la historia económica de la entidad. Es una de las ramas más antiguas debido a que está estrechamente ligada con la producción de tipo artesanal y ha fungido como soporte en la instalación de plantas industriales.

En lo correspondiente a su posición en el ámbito nacional, los datos del censo económico 2004 señalan que Jalisco representa el tercer centro productor de muebles en cuanto al número de unidades económicas, $8.62 \%$, así como en términos de la generación 
de valor agregado censal bruto, $13.75 \%$, y se constituye como el principal respecto al personal ocupado en el sector, $12.70 \%$. Ahora bien, en relación con la importancia que la industria mueblera tiene para el estado de Jalisco, esta abarca $7.42 \%$ respecto al número de unidades económicas de la industria manufacturera, y genera $6.10 \%$ del personal ocupado y $2.36 \%$ del valor agregado censal bruto. Asimismo, $87 \%$ de los productores están especializados en la fabricación de muebles para el hogar, clase 33712, aportando también el mayor número de personal ocupado, $81.84 \%$, y generando el mayor porcentaje de valor agregado censal bruto, $76.02 \%$ (ver cuadro siguiente).

Cuadro 1. Unidades económicas, personal ocupado y valor agregado censal bruto para la industria mueblera en el estado de Jalisco, 2004

\begin{tabular}{|c|c|c|c|c|c|c|c|c|}
\hline \multirow{2}{*}{$\begin{array}{c}\text { Sector, } \\
\text { Subsector, } \\
\text { Rama, Clase }\end{array}$} & \multirow{2}{*}{\multicolumn{2}{|c|}{ Descripción }} & \multicolumn{2}{|c|}{ Unidades económicas } & \multicolumn{2}{|c|}{ Población ocupada } & \multicolumn{2}{|c|}{ Valor agregado censal bruto } \\
\hline & & & abs. & rel. & abs. & rel. & $a b s$. & rel. \\
\hline $31-33$ & \multicolumn{2}{|c|}{ Industria manufacturera } & 24,742 & $100 \%$ & 325,887 & $100 \%$ & $\$ 64,085,656$ & $100 \%$ \\
\hline 337 & \multicolumn{2}{|c|}{$\begin{array}{l}\text { Fabricación de muebles y productos } \\
\text { relacionados }\end{array}$} & 1,836 & $7.42 \%$ & 19,884 & $6.10 \%$ & $\$ 1,511,028$ & $2.36 \%$ \\
\hline \multirow{3}{*}{3371} & \multicolumn{2}{|c|}{$\begin{array}{l}\text { Fabricación de muebles, excepto de } \\
\text { oficina y estantería }\end{array}$} & 1,711 & $93.19 \%$ & 17,678 & $88.91 \%$ & $\$ 1,243,422$ & $82.29 \%$ \\
\hline & 33711 & Fabricación de cocinas & 115 & $6.26 \%$ & 1,405 & $7.95 \%$ & $\$ 94,756$ & $6.27 \%$ \\
\hline & 33712 & $\begin{array}{l}\text { Fabricación de muebles, } \\
\text { excepto cocinas y } \\
\text { muebles de oficina } \\
\text { y estantería }\end{array}$ & 1,596 & $86.93 \%$ & 16,273 & $92.05 \%$ & $\$ 1,148,666$ & $76.02 \%$ \\
\hline \multirow{2}{*}{3372} & \multicolumn{2}{|c|}{$\begin{array}{c}\text { Fabricación de muebles de oficina y } \\
\text { estantería }\end{array}$} & 89 & $4.85 \%$ & 856 & $4.30 \%$ & $\$ 65,219$ & $4.32 \%$ \\
\hline & 33721 & $\begin{array}{l}\text { Fabricación de muebles } \\
\text { de oficina y estantería }\end{array}$ & 89 & $4.85 \%$ & 856 & $4.30 \%$ & $\$ 65,219$ & $4.32 \%$ \\
\hline \multirow{3}{*}{3379} & \multicolumn{2}{|c|}{$\begin{array}{l}\text { Fabricación de productos } \\
\text { relacionados con los muebles }\end{array}$} & 36 & $1.96 \%$ & 1,350 & $6.79 \%$ & $\$ 202,387$ & $13.39 \%$ \\
\hline & 33791 & Fabricación de colchones & 27 & $1.47 \%$ & 116 & $0.58 \%$ & $\$ 6,155$ & $0.41 \%$ \\
\hline & 33792 & $\begin{array}{l}\text { Fabricación de persianas } \\
\text { y cortineros }\end{array}$ & 9 & $0.49 \%$ & 1,234 & $6.21 \%$ & $\$ 196,232$ & $12.99 \%$ \\
\hline
\end{tabular}

Fuente: Elaboración propia con base en el Censo Económico 2004, INEGI. 
Respecto a la localización de los establecimientos productores, estos se encuentran diseminados en la mayoría de los municipios del estado, aunque es preponderante su presencia en aquellos que comprenden la Zona Metropolitana de Guadalajara, ZMG: Guadalajara, Zapopan, Tlaquepaque y Tonalá; así como en el municipio de Ocotlán.

Si se habla de la tendencia en la producción de muebles, esta se ha visto modificada en las últimas décadas: De la fabricación de colchones, muebles de consumo popular y muebles regionales de tipo rústico (Padilla 1988) se ha pasado, según las últimas encuestas de coyuntura, a la elaboración de muebles de madera para el hogar, $46 \%$ de la producción: de estilo contemporáneo, 51\%; clásico, 30\%, y rústico, 15\% (SEIJAL 2004a, 2004b y 2005). De hecho, la alta especialización en la fabricación de muebles para el hogar, clase 33712, se concentra en el municipio de Guadalajara (ver Cuadro 2).

Cuadro 2. Unidades económicas de la industria mueblera en Jalisco, clase de actividad 33712, según ubicación

\begin{tabular}{|l|r|r|}
\hline \multicolumn{1}{|c|}{ Ubicación } & \multicolumn{2}{|c|}{ Unidades económicas } \\
\hline Jalisco & 1596 & $86.93 \%$ \\
\hline Guadalajara & 425 & $26.63 \%$ \\
\hline Zapopan & 170 & $10.65 \%$ \\
\hline Poncitlán & 136 & $8.52 \%$ \\
\hline Tonalá & 133 & $8.33 \%$ \\
\hline Tlaquepaque & 130 & $8.15 \%$ \\
\hline Zacoalco de Torres & 62 & $3.88 \%$ \\
\hline Zapotlán El Grande & 28 & $1.75 \%$ \\
\hline Casimiro Castillo & 21 & $1.32 \%$ \\
\hline San Martín Hidalgo & 19 & $1.19 \%$ \\
\hline Sayula & 16 & $1.00 \%$ \\
\hline
\end{tabular}

Fuente: Elaboración propia con datos de SAIC 5.0, INEGI.

Por otra parte, las estadísticas históricas brindan información sobre la configuración de la industria en los últimos cuarenta años (ver Cuadro 3 y Gráfica 1): la tasa de cambio, 
permite observar los vaivenes de su integración, con un crecimiento explosivo a partir de los años ochenta y una caída drástica en los últimos años, producto principalmente de los problemas que han afectado su competitividad: falta de financiamientos, carencia de mano de obra, insuficiencia de abasto de insumos, y la oferta desleal por la importación de muebles procedentes de China. Aunado a lo anterior, este sector productivo presenta una baja tasa de exportación, $8 \%,{ }^{3}$ además de que se considera como una actividad productiva que incorpora poco avance tecnológico y escaso diseño.

Cuadro 3. Número de establecimientos y personal ocupado total, en la industria mueblera en Jalisco

\begin{tabular}{|c|c|c|c|c|}
\hline \multirow{2}{*}{ Año } & \multicolumn{2}{|c|}{ Núm. de establecimientos } & \multicolumn{2}{c|}{ Personal ocupado total } \\
\cline { 2 - 5 } & & & & \\
& Núm. & Tasa de cambio & Núm. & Tasa de cambio \\
\hline 1966 & 109 & - & N. D. & - \\
\hline 1971 & 229 & $110.09 \%$ & 712 & - \\
\hline 1976 & 267 & $16.59 \%$ & 4,356 & $-60.35 \%$ \\
\hline 1981 & 241 & $-9.74 \%$ & 1,727 & $320.03 \%$ \\
\hline 1989 & 719 & $198.34 \%$ & 7,254 & $43.25 \%$ \\
\hline 1994 & 1,405 & $95.41 \%$ & 10,391 & $61.39 \%$ \\
\hline 1999 & 2,308 & $64.27 \%$ & 16,770 & $18.57 \%$ \\
\hline 2004 & 1,836 & $-20.45 \%$ & 19,884 & \\
\hline
\end{tabular}

Fuente: Elaboración propia con base en los censos económicos, INEGI, varios años. 
Gráfica 1

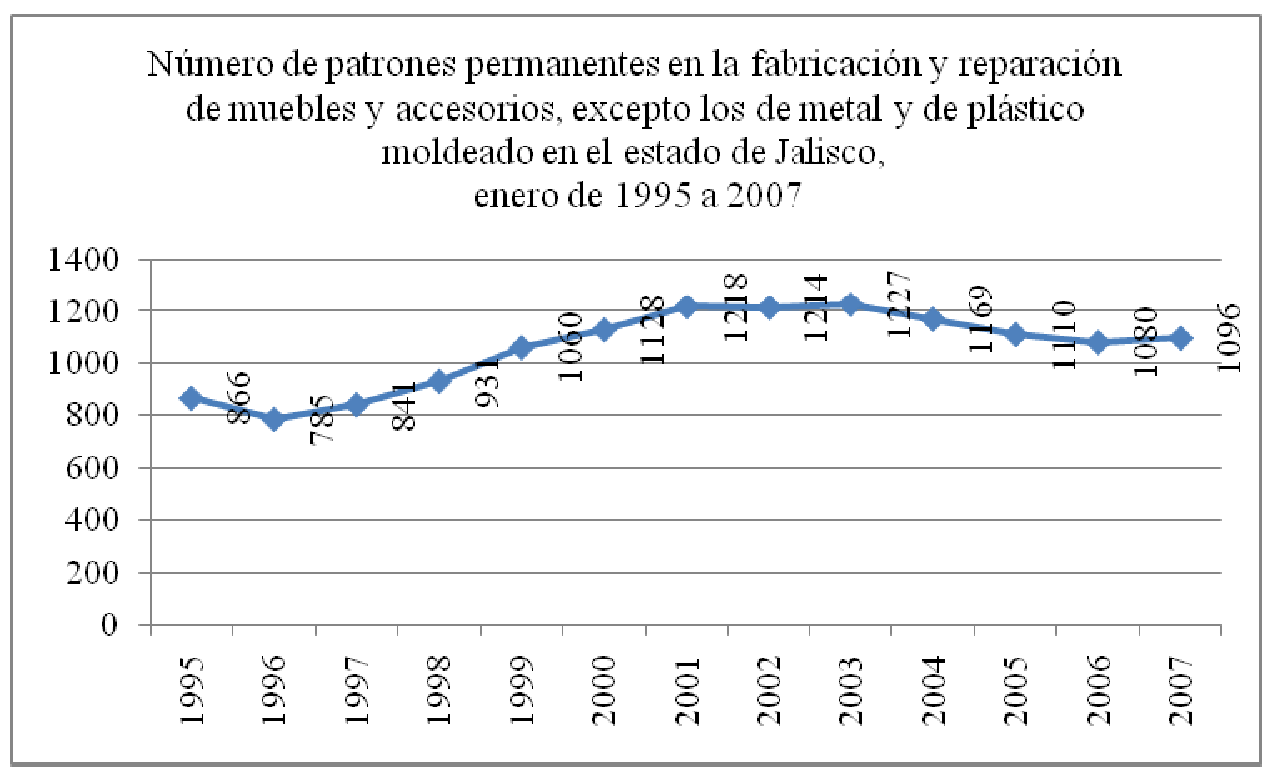

Fuente: Elaboración propia con base en SEIJAL y la base de datos proporcionados por IMSS.

La explicación a la trayectoria de crecimiento de la industria mueblera jalisciense en los años ochenta y noventa coincide con el planteamiento que hace Ruiz (2002a), quien sostiene que la expansión empresarial en los años noventa en México, analizada por especialización productiva en el sector manufacturero, se dio en los sectores de alimentos, bebidas y tabaco, seguidos por los de las industrias de maquinaria y equipo, y luego las dedicadas a la fabricación de muebles de madera y a la producción de prendas de vestir. Esta expansión en el ámbito territorial se concentró en el área central del país con 17.3\% del total, y en estados como Jalisco que reporta $8.42 \%$ de nuevas empresas en el año 2000, fundamentalmente de micro empresas (Ruiz 2002a; ver Cuadro 4). 
Cuadro 4. La expansión empresarial en Jalisco (número de nuevas empresas), 2000

\begin{tabular}{|c|c|c|c|c|c|}
\hline \multirow[b]{2}{*}{ Estado } & \multirow[b]{2}{*}{ Total } & \multicolumn{4}{|c|}{ Establecimiento } \\
\hline & & $\begin{array}{c}0-15 \\
\text { Micro }\end{array}$ & $\begin{array}{c}\text { 16-100 } \\
\text { Pequeño }\end{array}$ & $\begin{array}{l}101-250 \\
\text { Mediano }\end{array}$ & $\begin{array}{c}251 \text { o más } \\
\text { Grande }\end{array}$ \\
\hline \multirow{2}{*}{ Nacional } & 931,813 & 903,304 & 23,603 & 3,035 & 1,813 \\
\hline & $-100 \%$ & $-96.94 \%$ & $-2.53 \%$ & $-0.33 \%$ & $-0.19 \%$ \\
\hline \multirow{2}{*}{ Jalisco } & 78,493 & 75,707 & 2,403 & 255 & 128 \\
\hline & $-8.42 \%$ & $-96.45 \%$ & $-3.06 \%$ & $-0.32 \%$ & $-0.16 \%$ \\
\hline
\end{tabular}

Fuente: Elaboración propia con base en información presentada en Ruiz 2002a.

En el análisis que realiza el autor destacan para este caso los datos presentados referentes a las industrias de la madera y productos de la madera, incluyendo muebles; este tipo de industrias experimentaron $14.1 \%$ de expansión en la industria manufacturera. Sin embargo, la elevada presencia de micro empresas en la formación de nuevas empresas denota una elevada necesidad de autoempleo, con el fin de subsistir en una economía que presenta baja generación de empleo formal, y el impacto de la expansión empresarial en sectores con bajo perfil tecnológico, que da como resultado bajos niveles de productividad empresarial y de valor agregado.

Otra de las características de la industria mueblera en Jalisco es su elevada dependencia del mercado local: la producción se ha dirigido, considerando su participación promedio de los últimos años, en 58\% al mercado nacional, $16 \%$ al regional, y $18 \%$ al local (SEIJAL-CIMEJAL 2002a, 2002b y 2003; SEIJAL 2004a, 2004b y 2005). Esta particularidad es relevante cuando se analiza la empresarialidad, pues como lo establece Vigo (2004) la posibilidad de colocar inicialmente la producción en el mercado local adquiere gran importancia para el nacimiento de nuevas empresas, debido a que la demanda 
potencial inmediata de la nueva organización es en su mayor parte local. Sin embargo, el mantener una industria territorializada, ${ }^{4}$ es decir, dependiente del mercado nacional, que además se siente «cómoda» en dicho mercado, limita la búsqueda de oportunidades de negocios fuera del propio mercado. A su vez, en la medida en que la producción añadida por las empresas de reciente creación se destine al mercado local, los rasgos de la estructura manufacturera que determinan la entrada, supervivencia y crecimiento de las firmas, se mantienen en el ámbito regional (Viego 2004).

Por otra parte, como fue expuesto en la sección anterior, las instituciones juegan un papel esencial en la conformación del entorno que favorece la empresarialidad; en el caso de la industria mueblera jalisciense, las asociaciones empresariales han jugado un papel destacado no solo en la promoción del sector, también en la conformación de un entorno propicio para el desarrollo de los negocios. Entre las principales se tiene la presencia de la Asociación de Fabricantes de Muebles del Estado de Jalisco, AFAMJAL, fundada en 1979, que reporta la filiación de 88 socios; la Cámara de la Industria Mueblera del Estado de Jalisco, CIMEJAL, establecida en 1999, y la Asociación de Fabricantes de Muebles de Ocotlán, AFAMO, constituida en 1996, que actualmente cuenta con 66 socios activos. ${ }^{5}$

Por su parte, en términos de los organismos gubernamentales en el estado de Jalisco que favorecen la innovación, encontramos principalmente al Consejo Estatal de Ciencia y Tecnología, COECYTJAL, mismo que tiene como objetivo impulsar, fomentar, coordinar y coadyuvar en el desarrollo de las acciones públicas y privadas relacionadas con el avance de la ciencia y la tecnología en el estado, y en donde se han apoyado proyectos como la creación del Centro Articulador del Sector Mueblero de Jalisco, titulado por AFAMJAL. Así también existe el Instituto Jalisciense de la Calidad, que tiene como propósito la 
difusión de la cultura de «calidad» y la asistencia técnica y capacitación para la mejora de los procesos (Cuadro 5).

Cuadro 5. Entramado institucional y tipos de apoyo en la industria mueblera en Jalisco

\begin{tabular}{|l|l|l|}
\hline \multicolumn{2}{|c|}{ Institución } & \multicolumn{1}{c|}{ Tipo de apoyo } \\
\hline \multirow{2}{*}{$\begin{array}{l}\text { Cámaras y organizaciones } \\
\text { empresariales }\end{array}$} & $\begin{array}{l}\text { CIMEJAL } \\
\text { AFAMJAL }\end{array}$ & $\begin{array}{l}\text { Organizan la Expo Muebles Internacional, } \\
\text { edición de verano e invierno }\end{array}$ \\
\cline { 2 - 3 } Organismos gubernamentales & AFAMO & Organiza Expo Ocotlán Mueblera \\
\hline & COECYTJAL & $\begin{array}{l}\text { Impulsa, fomenta, coordina y coadyuva en las } \\
\text { acciones de ciencia y tecnología. } \\
\text { Apoya el proyecto Centro articulador del sector } \\
\text { mueblero de Jalisco }\end{array}$ \\
\cline { 2 - 3 } & $\begin{array}{l}\text { Instituto Jalisciense } \\
\text { de la Calidad }\end{array}$ & $\begin{array}{l}\text { Difunde la cultura de la calidad, provee de } \\
\text { asistencia técnica y capacitación en la mejora de } \\
\text { procesos }\end{array}$ \\
\cline { 2 - 3 } & SEPROE & \begin{tabular}{l} 
Promueve la cadena de la industria mueblera \\
\hline
\end{tabular} \\
\hline
\end{tabular}

Fuente: Lozano 2007.

Dentro de las iniciativas propuestas por las asociaciones de fabricantes para la integración de la cadena productiva madera-muebles y la mejora de la competitividad del sector, se encontró la formación del Centro de Investigación y Desarrollo de la Industria Mueblera, CIDIM, cuyo objetivo fue que las empresas pertenecientes al sector mueblero pudieran realizar pruebas de todos los insumos que utilizaban para la fabricación de muebles o de diferentes piezas y partes, con el fin de alcanzar los estándares de calidad exigidos por el mercado interno o las normas internacionales. Este proyecto contemplaba la participación de socios tanto de AFAMJAL como de CIMEJAL. Lamentablemente no se implementó en virtud de que «no respondía a las necesidades de las empresas» ${ }^{6}$, por lo que no reportó participación empresarial.

Actualmente, AFAMJAL ha puesto en marcha un Programa Integral de Desarrollo, PIDE, que tiene como objetivo básico el desarrollar la industria del mueble incrementando sus ventas, su productividad, sus márgenes de utilidad, y el valor agregado en sus productos 
mediante tecnología y diseño, a través del establecimiento de proyectos a largo plazo que tienen que ver con la creación de un centro tecnológico de madera que contempla el diseño y la innovación de muebles, así como un centro de formación (AFAMJAL 2007).

En el caso de Ocotlán, no obstante que AFAMO reporta en su página web que las empresas asociadas tienen vínculos con el Centro Universitario de la Ciénaga de la Universidad de Guadalajara, el Instituto Tecnológico de Ocotlán y el Centro de Capacitación para el Trabajo Industrial, CECATI; un estudio de la Secretaría de Economía (2006) indica relaciones nulas o débiles principalmente para las micro y pequeñas empresas (ver Cuadro 6).

Cuadro 6. Relaciones identificadas por tamaño de empresa en la industria mueblera de Ocotlán, Jalisco

\begin{tabular}{|c|c|c|c|c|c|c|}
\hline Empresa & $\begin{array}{c}\text { Otras } \\
\text { empresas }\end{array}$ & $\begin{array}{c}\text { Instituciones } \\
\text { educativas }\end{array}$ & $\begin{array}{c}\text { Instituciones } \\
\text { de gobierno }\end{array}$ & $I+D$ & $\begin{array}{c}\text { Centros de } \\
\text { servicio } \\
\text { especializado }\end{array}$ & Proveedores \\
\hline Micro & Nula & Nula & Nula & Nula & Nula & Nula \\
\hline Pequeña & Nula & Nula & Débil & Nula & Nula & Nula \\
\hline Mediana & Débil & Nula & Débil & Nula & Nula & Fuerte \\
\hline Grande & Débil & Nula & Débil & Nula & Nula & Fuerte \\
\hline
\end{tabular}

Fuente: Secretaría de Economía 2006.

Por su parte los organismos gubernamentales, como la Secretaría de Promoción Económica del Estado de Jalisco, tienen detectadas tanto las áreas de oportunidad como las debilidades y amenazas del sector. El estudio «Programa para la competitividad de la industria del mueble en Jalisco» lo denota, al referir que según los resultados de entrevistas en profundidad realizadas por COECYTJAL, los empresarios creen que sus empresas se volverían más competitivas si pudieran automatizar procesos, para lo que se requieren créditos con los que adquirir tecnología, mejorar la calidad e introducir nuevos productos, 
reducir su precio de venta, dar capacitación en áreas de servicio y atención, hacer más eficiente la venta y reparto e impulsar nuevos nichos de mercado (SEPROE sin fecha; ver Cuadro 7).

Cuadro 7. Factores para mejorar la competitividad de las empresas de la industria mueblera en Jalisco

\begin{tabular}{|c|c|}
\hline \multicolumn{2}{|r|}{ Factores para mejorar la competitividad de su empresa } \\
\hline Infraestructura & $\begin{array}{l}\text { - Automatización } \\
\text { - Tecnología } \\
\text { - Fomento y crédito que impulse la tecnificación } \\
\text { - Más maquinaria de corte }\end{array}$ \\
\hline Producto & $\begin{array}{l}\text { - } \text { Eficaz producción } \\
\text { - Productos nuevos } \\
\text { - } \text { Calidad del producto y materiales } \\
\text { - } \text { Bajando los costos } \\
\text { - Mejorando los precios } \\
\text { - Manejando más materiales } \\
\end{array}$ \\
\hline Servicio & $\begin{array}{l}\text { - Buen desempeño de todas las actividades internas en todas las áreas } \\
\text { - Capacitación constante, sobre todo en las áreas de servicios y atención } \\
\text { - Eficaz reparto } \\
\text { - Eficaz venta } \\
\text { - Apertura hacia el mercado } \\
\text { - Satisfacción de la demanda } \\
\text { - Informarse sobre las necesidades del cliente } \\
\text { - Servicio al cliente } \\
\text { - Impulsar el desarrollo de nuevos nichos de mercado } \\
\text { - Hacer un estudio de mercado para que sepan qué van a hacer } \\
\text { - Mejorando la situación estándar de los precios que se puedan establecer de manera } \\
\text { general; que haya más lealtad en este tipo de situaciones }\end{array}$ \\
\hline
\end{tabular}

Fuente: SEPROE, sin fecha.

A partir de lo anterior el programa de competitividad propone la ejecución de proyectos en siete áreas estratégicas y dispone responsabilidad conjunta de los actores institucionales, asociaciones empresariales y organismos gubernamentales, además de acciones particulares en los ámbitos macro, meso y micro, mediante la creación de un Centro de Articulación Productiva y Vinculación Empresarial que priorice, coordine, promueva y evalúe las acciones y los resultados de la cadena productiva (ver Cuadro 8). 
Cuadro 8. Estrategias y acciones en el «Programa para la competitividad de la industria del mueble en Jalisco»

\begin{tabular}{|l|l|l|l|}
\hline \multicolumn{1}{|c|}{ Estrategia } & \multicolumn{1}{|c|}{$\begin{array}{c}\text { Responsable } \\
\text { Sector Privado }\end{array}$} & \multicolumn{1}{c|}{$\begin{array}{c}\text { Responsable } \\
\text { Sector Público }\end{array}$} & \multicolumn{1}{c|}{$\begin{array}{c}\text { Avances } \\
2005\end{array}$} \\
\hline $\begin{array}{l}\text { Sistema de información e } \\
\text { inteligencia de mercado }\end{array}$ & AFAMO & SEIJAL & $\begin{array}{l}\text { Diseño de página Web, info. de } \\
\text { eventos, apoyos }\end{array}$ \\
\hline $\begin{array}{l}\text { Innovación, tecnología y } \\
\text { logística }\end{array}$ & CIMEJAL & COECYTJAL & Diagnóstico a treinta empresas \\
\hline $\begin{array}{l}\text { Financiamiento } \\
\text { AFAMO } \\
\text { interno }\end{array}$ & DGPIIF & $\begin{array}{l}\text { Esquemas de factoraje con ING } \\
\text { Factoring, financiamiento HSBC } \\
\text { con garantías líquidas de S.E. y } \\
\text { NAFIN }\end{array}$ \\
\hline $\begin{array}{l}\text { Normalización certificación y } \\
\text { capacitación }\end{array}$ & CIMEJAL & INJAC & $\begin{array}{l}\text { Proyecto de desarrollo } \\
\text { proveedores }\end{array}$ \\
\hline Integración productiva & CAREINTRA & DGS & $\begin{array}{l}\text { Diseño de proyecto piloto para } \\
\text { intervención en treinta empresas }\end{array}$ \\
\hline Promoción exportaciones & AFAMJAL & JALTRADE/CIDUE & $\begin{array}{l}\text { Integradoras (3) } \\
\text { Estudio de mercado en Texas. } \\
\text { compradores a Expo Mueble }\end{array}$ \\
\hline
\end{tabular}

Fuente: SEPROE, sin fecha.

Por lo anterior cabe preguntar, ¿en qué tipo de políticas se están centrando las instituciones para promover la industria mueblera en Jalisco? Las acciones denotan que las estrategias se dirigen a tener una respuesta rápida ante las condiciones del entorno que permita sobre todo subsistir y aprender a adaptarse principalmente a través de: 1) fomentar la capacidad exportadora, 2) el desarrollo de redes empresariales —integradoras—, a partir de fortalecer la cadena productiva mediante el desarrollo de proveedores y el surgimiento de agrupamientos empresariales, y 3) una estrategia de competencia mediante la innovación y el mejoramiento de la calidad.

Cabe destacar dentro del programa de competitividad las acciones en torno al financiamiento, debido a que es un factor que de manera reiterada se señala como obstáculo que minimiza la oferta en el sector. En este caso, el programa de competitividad prevé acciones que permiten a las empresas acceder a créditos avalados por un comité sectorial, 
participar en el Fondo $\mathrm{PYME}^{7}$ — programa federal—, así como aprovechar mecanismos como el factoraje ${ }^{8}$ con Nacional Financiera.

\section{REFLEXIONES FINALES}

De los principales componentes del desarrollo local se pueden comentar la dimensión económica y el potenciamiento de los recursos locales, a partir del desarrollo empresarial y de las relaciones interempresariales que se entretejen entre los actores empresariales, así como con las organizaciones públicas y privadas de promoción y fomento.

La empresarialidad es uno de los factores que promueven la generación de procesos de desarrollo local, en virtud de que no solo se trata de la creación de nuevas empresas sino que también contempla el desarrollo de ideas y proyectos de negocios que amplían el tejido productivo y la innovación.

El caso de la industria mueblera en Jalisco es posible analizarlo desde el desarrollo local y como un sistema productivo local, en primer término por sus características intrínsecas: predominio de micro y pequeñas empresas, su concentración en la zona metropolitana de Guadalajara y Ocotlán, su identidad y tradición productiva en la entidad, la elevada generación de empleo y la formación de un mercado de trabajo especializado, sobre todo en la fabricación de muebles para el hogar, entre los principales.

Sin embargo, las circunstancias actuales evidencian los grandes retos que les presenta la globalización a los sectores tradicionales como el del mueble. En este sentido, es una realidad que la base empresarial de la industria mueblera en Jalisco se ha reducido en los últimos años, por tanto el fomento a la empresarialidad está asociado con la búsqueda de una mayor competitividad que revierta la tendencia de crecimiento negativo, 
es decir, centrada en la regeneración de los tejidos socioproductivos y en el aporte de nuevas ideas e innovación.

La gran ventaja que presenta el sector es el interés conjunto de las organizaciones gremiales y los actores gubernamentales, no obstante la baja tasa de agremiación. En este caso, las asociaciones empresariales y cámara respectiva han impulsado a partir de los distintos proyectos de articulación productiva iniciativas para la construcción de un entorno competitivo que permita un mayor desarrollo del sector, y la creación de la confianza necesaria para su crecimiento en el estado de Jalisco. En este sentido, como lo afirma Kantis (2004b), una clave para la sostenibilidad del desarrollo empresarial es el compromiso compartido, fundamental para despertar las capacidades locales y operar de manera descentralizada bajo esquemas que garanticen la coordinación y la racionalidad de los esfuerzos.

Desde esta perspectiva un importante reto para la industria mueblera es el fortalecimiento de la confianza al interior del sector, para que se materialice en el incremento en la filiación de las organizaciones empresariales así como en la construcción de un mayor número de relaciones de cooperación y de redes empresariales.

Desde este mismo punto de vista, el fomento a la empresarialidad y un incremento de la competitividad de la industria mueblera de Jalisco también requiere de una mayor vinculación con las universidades localizadas en el estado: la ejecución de proyectos y programas de investigación y desarrollo, la dotación de servicios especializados, incubadoras de empresas, trasferencia de tecnología y la capacitación, son algunas de las posibles interacciones que se pueden establecer y aprovechar. 
Por su parte, la estrategia de desarrollo empresarial de la industria mueblera de Jalisco, propuesta en el programa de competitividad, se ha centrado en combatir las debilidades y amenazas del sector a partir de la innovación, el fomento a la calidad, la promoción de las exportaciones y la integración de la cadena productiva, como camino de sobrevivencia y de adaptación a las condiciones que van planteando los rápidos cambios del entorno. No obstante, aún faltan por incorporar acciones que promuevan el aprendizaje y la capacitación a los empresarios locales. En el largo plazo no se contempla la acumulación regional de una masa crítica de empresarios que conforme un lenguaje común que permita el intercambio de experiencias y que, por tanto, posibilite la cooperación interempresarial como una manera de alimentar y fortalecer el desarrollo empresarial (Ruiz 2002b: 184).

Además, lo importante para su consecución será lograr que un número significativo de empresas fabricantes de muebles se inserten en las dinámicas señaladas en los proyectos, lo que generaría un sistema productivo local competitivo en los ámbitos nacional e internacional, que aprovecharía los recursos que le provee la interacción dada su concentración —economías externas- y que le permitiría en el largo plazo generar un ambiente para el nacimiento de nuevas empresas viables; esto es, que el proyecto no muera en el corto plazo, que posea características competitivas de orden superior, centradas en la innovación, la calidad y la diferenciación de sus productos, más que en el precio de los mismos.

Por ahora es necesario esperar los resultados que arroje la puesta en marcha del Programa de competitividad de la industria del mueble en Jalisco para definir el impacto 
que tiene sobre todo en la base empresarial de micro y pequeños empresarios, que constituyen más de 95\% del total de las empresas del sector. 


\section{BIBLIOGRAFÍA}

Alburquerque, Francisco, 2005, «Espacio, territorio e instituciones de desarrollo económico local». En Desarrollo Local, textos cardinales, pp. 90-107, compilado por Andrés Solari y Jorge Martínez. Facultad de Economía Vasco de Quiroga, División de Estudios de Posgrado, Universidad Michoacana de San Nicolás de Hidalgo, México.

Angelelli, Pablo Javier y Juan José Llisterri, 2003, «El BID y la promoción de la empresarialidad: Lecciones aprendidas y recomendaciones para nuevos programas». Washington, D. C.

Arocena, José, 1995, El Desarrollo Local: Un Desafío Contemporáneo. Centro Latinoamericano de Economía Humana, Universidad Católica de Uruguay, Editorial Nueva Sociedad, Venezuela.

Asociación de Fabricantes de Muebles de Jalisco, A. C., AFAMJAL, 2007, «Programa Integral de Desarrollo». Inédito.

Carrillo, Emilio, 2002, Desarrollo local: Nuevas perspectivas. Consejería de Gobernación, Dirección General de Administración Local de la Junta de Andalucía, España.

Centro de Estudios Estratégicos para el Desarrollo, CEED, 2006, Jalisco. Matriz InsumoProducto 1996. Universidad de Guadalajara, México.

Consejo Estatal de Ciencia y Tecnología, Jalisco, COECYTJAL, 2004, Programa Estatal de Ciencia y Tecnología de Jalisco. En http://coecyt.jalisco.gob.mx/pecytjal.htm [consulta: diciembre de 2006]. 
Cortelesse, Claudio, 1993, «Competitividad de los sistemas productivos y las empresas pequeñas y medianas: Campo para la cooperación internacional». Revista Comercio Exterior, vol. 43, núm. 6, pp. 519-524. Banco Nacional de Comercio Exterior, S. N. C., México.

Instituto Nacional de Estadística, Geografía e Informática, INEGI, 2004, «XVI Censo Industrial». Censos Económicos. INEGI, México.

Kantis, Hugo, 2002, Empresarialidad en economías emergentes: Creación y desarrollo de nuevas empresas en América Latina y el Este de Asia. Banco Interamericano de Desarrollo.

2004a, «Un enfoque sistémico de la creación de empresas». En Desarrollo Emprendedor. América Latina y la Experiencia Internacional, pp. 265-281, editado por Hugo Kantis, con la colaboración de Pablo Angelelli y Virginia Moori, pp. 2134. Banco Interamericano de Desarrollo, Fundes Internacional, Colombia.

2004b, «Principales lecciones para América Latina». En Desarrollo Emprendedor. América Latina y la Experiencia Internacional, editado por Hugo Kantis, con la colaboración de Pablo Angelelli y Virginia Moori. Banco Interamericano de Desarrollo, Fundes Internacional, Colombia.

Lozano, Katia Magdalena, 2007, «Los sistemas productivos locales y la importancia de la innovación en la construcción del territorio: El caso de la industria mueblera en Jalisco, México». En Sistemas Productivos Locales en México. Tipología desde la Perspectiva Europea, pp. 189-213, editado por Xavier Paunero y Leonel Corona. Documenta Universitaria, Universidad de Girona, España. 
Lozano, Katia Magdalena y Pedro Méndez, 2002, «La industria del calzado en la Zona Metropolitana de Guadalajara, desde la óptica del desarrollo local». En Desarrollo local, innovaciones y redes empresariales, pp. 39-51, coordinado por Andrés Solari. Universidad Michoacana de San Nicolás de Hidalgo, México.

Méndez, Ricardo, 2000, «Procesos de innovación en el territorio: Los medios innovadores». En Innovación, Pequeña Empresa y Desarrollo Local en España, pp. 23-59, coordinado por José Luis Alonso y Ricardo Méndez. Civitas Ediciones, S. L., Madrid.

Padilla Dieste, Cristina, 1988, «La pequeña y mediana industria (1940-1980)». En Crecimiento industrial y manufacturero, 1940-1980, pp. 57-201, coordinado por Rogelio Luna y otros. Jalisco desde la Revolución, vol. XIII. Gobierno del Estado de Jalisco, Universidad de Guadalajara, México.

Ruiz, Clemente, 2002a, «Empresarialidad y desarrollo económico en México. El caso de las Mipymes». En Desarrollo Empresarial en América Latina, pp. 109-145, coordinado por Clemente Ruiz Durán. Nacional Financiera, UNAM, México.

2002b, «Adaptación y éxito de las pequeñas y medianas empresas a procesos de apertura». En Desarrollo Empresarial en América Latina, pp. 109-145, coordinado por Clemente Ruiz. Nacional Financiera, UNAM, México.

2005, Dimensión Territorial del Desarrollo Económico en México. Segunda Edición. Facultad de Economía-UNAM, México.

Secretaría de Economía, 2006, Intervención sobre el caso de Ocotlán, Jalisco. Contrastes con Italia y áreas de oportunidad. En http://www.economia.gob.mx/pics/p/p2757/CasoJalisco-Mueble.pdf [consulta: octubre de 2006]. 
Secretaría de Promoción Económica del Estado de Jalisco, SEPROE, s.a., «Programa para la Competitividad de la Industria del Mueble en Jalisco». Gobierno del Estado de Jalisco, México.

Sforzi, Fabio, 2001, «La Teoría Marshalliana para explicar el Desarrollo Local». En Manual de Desarrollo Local, pp. 13-32, editado por Fermín Rodríguez. Ediciones TREA, Madrid.

Sistema Estatal de Información Jalisco, SEIJAL, 2004a, Encuesta de Coyuntura. 1er. Semestre de 2004. En http://seijal.jalisco.gob.mx/estudios/mueblera /1erSem2004.pdf [consulta: diciembre de 2007].

2004b, Encuesta de Coyuntura. 2do. Semestre de 2004. En http://seijal.jalisco.gob.mx/estudios/mueblera/2doSem2004.pdf [consulta: diciembre de 2007].

2005, Encuesta de Coyuntura. ler. Semestre de 2005. En http://seijal.jalisco.gob.mx/estudios/mueblera/1erSem2005.pdf [consulta: diciembre de 2007].

Sistema Estatal de Información Jalisco y Cámara de la Industria Mueblera del Estado de Jalisco, SEIJAL-CIMEJAL, 2002a, Encuesta de Coyuntura. 1er. Semestre de 2002. En http://seijal.jalisco.gob.mx/estudios/mueblera/1erSem2002.pdf [consulta: diciembre de 2007].

2002b, Encuesta de Coyuntura. 2do. Semestre de 2002. En http://seijal.jalisco.gob.mx/estudios/mueblera/2doSem2002.pdf [consulta: diciembre de 2007]. 
2003, Encuesta de Coyuntura. ler. Semestre de 2003. En http://seijal.jalisco.gob.mx/estudios/mueblera/1erSem2003.pdf [consulta: diciembre de 2007].

Stumpo, Giovanni, 2004, «Articulación Productiva y Pequeñas y Medianas Empresas: Reflexiones a partir de Algunos Estudios de Caso en América Latina». En Pequeñas y Medianas Empresas y Eficiencia Colectiva. Estudios de Caso en América Latina, pp. 11-30, coordinado por Marco Dini y Giovanni Stumpo. CEPAL, Siglo XXI Editores, México.

Vachon, Bernard, 2001, El Desarrollo Local, Teoría y Práctica. Reintroducir lo humano en la lógica del desarrollo. Ediciones TREA, Madrid.

Vázquez, Antonio, 1988, Desarrollo Local. Una Estrategia de Creación de Empleo. Ediciones Pirámide, Madrid.

1993, Política Económica Local. La Respuesta de las Ciudades a los Desafíos del Ajuste Productivo. Ediciones Pirámide, Madrid.

1999, Desarrollo, Redes e Innovación. Lecciones sobre Desarrollo Endógeno. Ediciones Pirámide, Madrid.

2001, «La Política de Desarrollo Económico Local en Europa». En Manual de Desarrollo Local, pp. 42-60, editado por Fermín Rodríguez. Ediciones TREA, Madrid.

2005, «Desarrollo económico local y descentralización». En Desarrollo Local, textos cardinales, pp. 33-68, compilado por Andrés Solari y Jorge Martínez. Facultad de Economía Vasco de Quiroga, División de Estudios de Posgrado, Universidad Michoacana de San Nicolás de Hidalgo, México. 
Viego, Valentina, 2004, «Empresarialidad e instituciones: dos nuevas perspectivas del análisis regional contemporáneo». Revista EURE, vol. XXX, núm. 90, pp. 41-63. Pontificia Universidad Católica de Chile, Facultad de Arquitectura, Diseño y Estudios Urbanos Instituto de Estudios Urbanos y Territoriales, Santiago de Chile. 


\section{Notas}

${ }^{1}$ Alburquerque precisa: «El desarrollo productivo no es solo una cuestión que dependa de las directrices e intervenciones del Estado, ni tampoco es resultado tan solo de las actividades empresariales privadas. Dicho desarrollo depende de cómo el conjunto de la comunidad organiza la producción social. De hecho, el logro de la eficiencia productiva y competitividad de las empresas privadas es función "sistémica" de todo un conjunto de actividades que costea la sociedad, como las educativas y de capacitación de los recursos humanos, de la salud, higiene y vivienda, las de la adecuada resolución de conflictos en el sistema judicial, y sobre todo, las ocupadas en garantizar la existencia de los recursos estratégicos o servicios avanzados a la producción en el triple frente de la información, la capacitación y el acceso a la financiación» (2005: 91).

${ }^{2}$ Las instituciones se definen como el conjunto de normas y acuerdos que se dan entre los actores, las organizaciones y los pueblos, para regular sus relaciones económicas, sociales y políticas. Se trata no solo de reglas formales, como las constituciones, las leyes y los instrumentos para aplicarlas, sino también de todas aquellas normas informales como las pautas de conducta, los códigos o las convenciones, y aquellas otras formas como los contratos, que condicionan el comportamiento de las empresas y de la población de un territorio (Vázquez 2005: 119).

${ }^{3}$ En el año 2000, la exportación superó 39.7 millones de dólares, siendo los principales destinos: Estados Unidos, 81.5\%; Emiratos Árabes, 3.34\%; Venezuela, 2.67\%, y Panamá, 2.14\% (COECYTJAL 2004: 94).

${ }^{4}$ Las evidencias de esta territorialización de la industria se muestran también a partir de los resultados de las transacciones totales de la Matriz de Insumo-Producto de Jalisco en 1990, donde se expresa que $47.88 \%$ de la demanda intermedia se hace por el mismo sector de la industria de la madera y productos de la madera, en tanto que la demanda final se hace en su mayor parte por el consumo privado, $41.86 \%$, ventas al resto del país — productos que no se quedan en el estado de Jalisco—, 37.97\%, y ratifica un porcentaje reducido de exportación $12.30 \%$ (CEED 2006).

${ }^{5}$ La ciudad de Ocotlán, además de la zona metropolitana de Guadalajara, se ha constituido desde la década de los ochenta del siglo pasado como uno de los principales sitios que alberga una importante aglomeración de empresas productoras de muebles. Actualmente, según cifras del censo económico 2004, se reporta la presencia de 243 empresas, $13.24 \%$ del total registrado para el estado de Jalisco. Las empresas se distinguen por fabricar muebles para el hogar de madera, de tablero de astilla y MDF.

${ }^{6}$ Entrevista con funcionarios de AFAMJAL, 5 de diciembre de 2006.

${ }^{7}$ El Fondo de Apoyo para la Micro, Pequeña y Mediana Empresa, FONDO PYME, es un instrumento que busca apoyar a las empresas, en particular a las de menor tamaño, y a los emprendedores, con el propósito de promover el desarrollo económico nacional a través del otorgamiento de apoyos de carácter temporal a programas y proyectos que fomenten la creación, desarrollo, consolidación, viabilidad, productividad, competitividad y sustentabilidad de las micro, pequeñas y medianas empresas.

${ }^{8}$ El concepto de factoraje se entiende como la venta de las cuentas por cobrar de una empresa, haciendo un descuento sobre el valor del documento a cambio de efectivo inmediato. El factoraje permite a la empresa convertir sus cuentas por cobrar en efectivo inmediato al concluir una venta de bienes o por servicios prestados.

Fecha de recepción: 31 de enero de 2008 Fecha de aceptación: 07 de abril de 2008 